\title{
Neural Networks
}

\author{
Patrick Smith \\ Office of Science, Student Undergraduate Laboratory Internship (SULI) \\ Stanford University \\ Stanford Linear Accelerator Center \\ Menlo Park, Califomia
}

August 14, 2003

Prepared in partial fulfillment of the requirements of the Office of Science, Department of Energy's Science Undergraduate Laboratory Internship under the direction of

Tony Johnson. 


\section{INTRODUCTION}

Physicists use large detectors to measure particles created in high-energy collisions at particle accelerators. These detectors typically produce signals indicating either where ionization occurs along the path of the particle, or where energy is deposited by the particle. The data produced by these signals is fed into pattern recognition programs to try to identify what particles were produced, and to measure the energy and direction of these particles. Ideally, there are many techniques used in this pattern recognition software. One technique, neural networks, is particularly suitable for identifying what type of particle caused by a set of energy deposits. Neural networks can derive meaning from complicated or imprecise data, extract patterns, and detect trends that are too complex to be noticed by either humans or other computer related processes.

To assist in the advancement of this technology, Physicists use a tool kit to experiment with several neural network techniques. The goal of this research is interface a neural network tool kit into Java Analysis Studio (JAS3), an application that allows data to be analyzed from any experiment. As the final result, a physicist will have the ability to train, test, and implement a neural network with the desired output while using JAS3 to analyze the results or output.

Before an implementation of a neural network can take place, a firm understanding of what a neural network is and how it works is beneficial. A neural network is an artificial representation of the human brain that tries to simulate the learning process [5]. It is also important to think of the word artificial in that definition as computer programs that use calculations during the learning process. In short, a neural network learns by representative examples.

Perhaps the easiest way to describe the way neural networks learn is to explain how the human brain functions. The human brain contains billions of neural cells that are responsible for 
processing information [2]. Each one of these cells acts as a simple processor. When individual cells interact with one another, the complex abilities of the brain are made possible. In neural networks, the input or data are processed by a propagation function that adds up the values of all the incoming data. The ending value is then compared with a threshold or specific value. The resulting value must exceed the activation function value in order to become output. The activation function is a mathematical function that a neuron uses to produce an output referring to its input value. [8] Figure 1 depicts this process. Neural networks usually have three components an input, a hidden, and an output. These layers create the end result of the neural network. A real world example is a child associating the word dog with a picture. The child says dog and simultaneously looks a picture of a dog. The input is the spoken word "dog", the hidden is the brain processing, and the output will be the category of the word dog based on the picture. This illustration describes how a neural network functions.

\section{MATERIALS AND METHODS}

Before the implementation of a neural network tool kit into JAS3, JAS3 and all the components had to be installed. An editor that was capable of the processes had to be obtained and downloaded successfully on the workstation. The neural network that we originally used was Java Object Oriented Neural Engine (JOONE). This editor would eventually have to be replaced as you will find out why in the conclusion of this paper.

There were several factors that were involved in choosing the editor. It had to be userfriendiy, free software, flexible and capable of handling the amount of data for our purpose. The tool-kit will be used by physicists who are analyzing their specific data, so the application must be easy to use and understand. An equally important factor is that the editor is written in Java 
programming language because the editor will be interfaced with JAS3, a Java based application. Many companies sell neural network software. These software packages are very expensive. For the purpose of our research there was no need for one of those software packages. A more general editor would be beneficial. An editor that was flexible was also important. An editor with functionality that includes graphs and many other easy to use features would improve usability. There are many editors via the World Wide Web that met our needs and were free to download along with open source code. Finally, the editor had to be able to handle the specified amount of data.

Searching for a neural network editor involved the exploration of the internet. Many networks were available, however; five met most of the qualifications. The Stuttgart Neural Network simulator met the qualifications by a researcher at SLAC on a previous project. NeuroSolutions met all the requirements except that it was not free; in fact it was quite expensive. Cortex also met all the requirements except that it was written in $\mathrm{C}++$. JOONE satisfied all requirements, so originally JOONE was chosen.

Now that the editor was chosen, understanding the components of it was important. JOONE a GUI editor used a graphical user interface that allows you to create, modify, and train a neural network. JOONE for the most part is very self explanatory and there is a tutorial that is somewhat helpful. In addition the creator of JOONE, Paolo Marrone, responded to questions concerning his editor that were unclear to us. Figure 2 shows the JOONE editor.

For this study we have used a neural network to identify colorimeter clusters in a possible linear collinear collider detector. In such a detector hundreds of particles are created in each collision, each in which deposits its energy into a set of calorimeter cells. Software groups the energy deposits into clusters, representing the energy deposited by a single particle. By 
measuring various properties of the cluster and feeding these measurements into a neural network we can attempt to estimate what type of particle created each cluster. To train the network we use simulated events, in which we already know which type of particle created each energy deposit. The data that we used in the neural net came from Gary Bower a SLAC physicist who created a neural network using a different editor. His training and validation data was theoretically valuable in a couple of instances. When training a neural network, the data should be trained on more than one net to contrast and compare results. Bower's data gave us that element for comparison. Also, we could use it to train in different modes just to see the varying outputs.

We constructed a neural network that included 3 layers. The input layer consisted of 15 , the hidden 25 , and the output 6 . That means that the data set, the first 15 numbers in a row would be the input and the last 6 would be the desired output according to the data combination of the input. The hidden layer varies. According to Kevin Swinger, the hidden layer value should be no more than twice the input. It is practical to experiment with the hidden layer value. When the layers where finished, another component was used to read the data into the network referred to as the file input. The component simply read the first 15 columns into the network. Another component called the teacher read the last 6 columns or desired results into the network. The 21 rows of data that we used to train our network was the form of binary numbers. The first 15 inputs were named: NE [0], NE [1], NE [2], NE [0]/NE [1], NE [1]/NE [2], firstL, lastL, length, firstDdiff, aveLE5, angsep*10000, aveLHits2, nhits, ClusEtot, and CE[2] was the quantities that described the clusters. The last 6 desired outputs were nngamma, nnchhad, nneuhad, nngammafrag, nnhadfrag, and nnnone. These quantities identified what type of cluster it was. Example, if the first 15 binary numbers describe the last 6 rows to be " 100000 " it 
would be considered a nngamma cluster. This is because "100000" = nngamma in binary, as "001000" = to a mneuhad cluster in binary. See table 1 for complete transiations. We set the initial learning rate $\eta$ (eta) and momentum terms $\alpha$ (alpha). Eta dictates the proportion of the calculated error that contributes to the weight change. Alpha relates to the size of the previous update for each weight. [8] Although we left the values to the editor default, the values were important to monitor and prevent over fitting the data. We then set the number of cycles (epochs) for the network to train the data to 10,000 . The network does the rest.

The purpose of training the net is to minimize the error which the network makes at each output unit over the entire data set. [8] So while training we looked for our error to move closer and closer to zero. It will never actually reach zero. If it did the network simply memorized the training data. So if the training error reached a predetermined target value, flattened out, or start to rise, training should be stopped. The last step was to read the output into a file for comparison. The output was read into ADA. AIDA histogram interfaces allowed for a simple and easy to interpret implementation of the output.

\section{RESULTS}

Table 1 represents sample data that was used to train the net. Each row represents a different data set. The first 15 was used as inputs the last 6 desired outputs. The input rows determined what the last 6 (desired output) should be. Table 2 is the validation data or actual data that represent the values of the data we train. Note that the training data input is not identical to the validation input. This is because we do not want to train the net to memorize the data; instead we want to teach the net how to categorize the data. So when training data, the error should continue to decrease as the epochs increase. 
We tried JOONE on the complex set of data with 15 inputs, but we were unable to get it reach our desired outcome. We studied theory on neural networks, contacted the author, and tried different neural configurations. The results were never successful. We then thought that the data we were using was complex, so we would try simpler data. That experiment did not work as well. See figure 3 for the graphical output that JOONE plotted. As you can notice in the plot, the error does not continue to decrease instead it fluctuates. We then decided to try another neural editor named CINN. We tested the same simple data set that we used with JOONE. The output looked much better, so we proceeded to test CJNN on the complex data set. Again, the output was much better. See figure 4 for the graphical representation of the data trained on CJNN. Notice that the error decreases as the epochs increase. In figure 5 we used AIDA for analysis of our results from training the net. The plot ino represents what desired data was used for training. OutO displays the results that we obtained after it was trained. If the OutO satisfactory it would closely resemble in 0 . In a closer analysis the bottom left and right plots out 0 when in $0==1$ and out 0 when $i n O==0$ if the net was working properly we would get all 1 in the left plot and all 0 in the right plot.

\section{DISCUSSION AND CONCLUSION}

Although the initial of creation and implementation of a neural net seemed simple, I learned that there are many factors that one must take into accord. We faced many obstacles while attempting to successfully construct the network. At one point during our research it seemed as when one barrier was crossed another barrier would be put up. The problems ranged from input data organization to the network not learning the data.

One of the first problems we encountered was that the JOONE editor required semi-colons between each data element. So we had to add semi-colons to 21 rows and over a 1000 columns 
of data. This was an easily fixable problem; nevertheless, it was time consuming to run a find and replace function. The JOONE editor included a tutorial that explained the basics of the functionalities of the editor. The problem with the tutorial was that it did not completely explain the application's capability. The training of the neural network was exhaustively consuming of the workstation. We would have to leave the computer on after we left the lab in the evening to allow the networks to complete training. At one point we analyzed trained data and realized the data was not being trained at all. For about 3 days we kept running tests but we continued to get flawed results.

Finally, my mentor and I concluded that JOONE, despite its great flexibility and functionality, was not working properly. We contacted Pathak Saurav a scientist from the University of Penn. He created a neural network call CJNN. This editor lacks the flexibility that JOONE, but when we ran the same data that we trained in JOONE it looked much better. We migrated away form JOONE and towards CJNN.

Before this research, I had little knowledge on this subject. During this research, I have learned a lot about how neural networks learn and we have reached farther towards our goal. With further work, Physicists will have an option to build a network and train data for their personal research. Because of the many problems that we encountered with JOONE and the 8 week time limited that I had, we were unable to interface a Neural Network editor completely into JAS3. While JOONE looks impressive and very flexible, we had limited success in making it complete the task we wanted. Although, CJNN is less flexible, it meets our need at the current time. We should continue to interface CJNN into JAS3. 


\begin{abstract}
AKNOWLEDGEMENTS
I would like to take this time to thank the Department of Energy for the opportunity to participate in the SLAC/SULI internship. Special Thanks go to my mentor Tony Johnson for an excellent project that has my interest. I thank Tony for aspiration and a new found dedication towards figuring out the unknowns. I also would like to thank Max Turri for all of his efforts of assisting me. Lastly I would like to thank my new friends for the fulfilling experience at SLAC/SULI.
\end{abstract}




\section{REFRENCES}

[1] "Artificial Neural Networks in High Energy Physics" HEP.

http://neuralnets.web.cern.ch/NeuralNets/nnwlnHep.htm!

[2] Grossberg, Steven, Neural Networks and Natural Intelligence. Cambridge: Fourth Printing (1989).

[3] "Overview of Artificial Intelligence" JeffHeaton. www.jeffheaton.com/ai/javaneural/ch1.ht

[4] "Neural networks at Work" ieeespectrumjune 1993.

http://www.ece.ogi.edu/ strom/papers/spectruml.PDF

[5] "Neural Networks with Java" Fachhochschule Regensburg. http://rfhs8012.fhregensburg.de/ saij39122/jfroehl/diplom/e-index.him!

[6] "Java Object Oriented Neural Engine"JOONE. http://www-ra.informatik.unituebingen.de/SNNS/

[7] "Stuttgart Neural Network Simulator" University of Stuttgart. http://www-ra.informatik.unituebingen.de/SNNS/

[8] Swinger, Kevin, Applying Neural Networks a Practical Guide. San Francisco: Morgan Kaufman Publishers, Inc (2001). 


\begin{tabular}{|c|c|c|c|c|c|c|c|c|c|c|c|c|c|c|}
\hline 1 & 2 & 3 & 4 & 5 & 6 & 7 & 8 & 9 & 10 & 11 & 12 & 13 & 14 & \\
\hline 0.25004 & 0.72558 & 0.86318 & 0.34461 & 0.84058 & 4.60000 & 6.00000 & 3.00000 & 1.66660 & 0.04296 & 5983.20000 & 4.00000 & 12.00000 & 0.25777 & 153.910 \\
\hline 0.03091 & 0.28449 & 0.31539 & 0.10864 & 0.90201 & 1.00000 & 3.00000 & 3.00000 & 0.66667 & 0.01058 & 3267.30000 & 1.33330 & 4.00000 & 0.06348 & 138.050 \\
\hline 0.30590 & 6.96750 & 7.09420 & 0.04390 & 0.98215 & 0.00000 & 20.00000 & 21.00000 & 0.04762 & 0.20617 & $613.380 n 0$ & 1.33330 & 99.00000 & 4.56750 & 189.720 \\
\hline 0.03992 & 0.16875 & 0.20822 & 0.23654 & 0.81045 & 22.00000 & 24.00000 & 3.00000 & 7.66660 & 0.01226 & 9718.50000 & 1.66660 & 5.00000 & 0.07353 & 12.165 \\
\hline 0.03703 & 0.43066 & 0.46769 & 008599 & 0.92082 & 3.00000 & 7.00000 & 5.00000 & 0.80000 & 0.01105 & 4.53240 & 1.33330 & 6.00000 & 0.06632 & $10.50 \mathrm{t}$ \\
\hline 0.25247 & 38.60200 & 38.70900 & 0.00654 & 0.99724 & 28.00000 & 30.00000 & 3.00000 & 9.66660 & 0.03913 & 888.43000 & 3.33330 & 10.00000 & 0.23480 & -0.682 \\
\hline 0.11539 & 0.63563 & 0.64976 & 0.18153 & 0.97824 & 0.00000 & 6.00000 & 7.00000 & 0.14286 & 0.07644 & 1385.40000 & 2.00000 & 20.00000 & 0.47050 & -58.409 \\
\hline 0.06044 & 0.15082 & 0.19019 & 0.40073 & 0.79303 & 1.00000 & 2.00000 & 2.00000 & 1.00000 & 0.02505 & 12147.00000 & 1.66660 & 5.00000 & 0.15032 & -89.196 \\
\hline 0.41007 & 2.10940 & 2.18130 & 0.19440 & 0.96707 & 1.00000 & 10.00000 & 10.00000 & 0.20000 & 0.09010 & 1870.00000 & 3.33330 & 30.00000 & 0.68658 & 187.520 \\
\hline 0.33440 & 0.48850 & 0.49750 & 0.68455 & 0.98191 & 7.00000 & 11.00000 & 5.00000 & 1.60000 & 0.05257 & 13852.00000 & 4.00000 & 18.00000 & 0.31544 & 27.766 \\
\hline 0.03400 & 0.12319 & 0.13931 & 0.27599 & 0.88434 & 1.00000 & 2.00000 & 2,00000 & 1.00000 & 0.01126 & 12805.00000 & 1.66660 & 5.00000 & 0.06757 & -18.785 \\
\hline 0.17292 & 2.28410 & 2.33290 & 0.07571 & 0.97907 & 0.00000 & 11.00000 & 12.00000 & 0.08333 & 0.08613 & 358.28000 & 2.00000 & 37,00000 & 0.87463 & 41.148 \\
\hline 0.48116 & 3.76840 & 3.85420 & 0.12768 & 0.97775 & 1.00000 & 21.00000 & $23.0 \times 000$ & 0.09524 & 0.11356 & 856.09000 & 1.00000 & 154.00000 & 6.28410 & 11.511 \\
\hline 0.18082 & 0.35489 & 0.50208 & 0.50952 & 0.70683 & 22.00000 & 24.00000 & 3.00000 & 7.66660 & 0.03087 & 15327.00000 & 3.00000 & 9.00000 & 0.18519 & 12.360 \\
\hline 0.52078 & 2.91620 & 3.14000 & 0.17858 & 0.92874 & 1.00000 & 15.00000 & 15.00000 & 0.13333 & 0.25838 & 1055.50000 & 1.33330 & 91.00000 & 3.65650 & 31.846 \\
\hline 0.60028 & 3.80650 & 3.81770 & 0.15770 & 0.99707 & 0.00000 & 15.00000 & 16.00000 & 0.06250 & 0.28897 & 635.15000 & 2.33330 & 86.00000 & 4.12300 & 188.540 \\
\hline 0.08455 & 1.86600 & 1.87020 & 0.04531 & 0.99776 & 2.00000 & 14.00000 & 13.00000 & 0.23077 & 0.15618 & 730.42000 & 1.66660 & 68.00000 & 1.94920 & 189.300 \\
\hline 0.40779 & 4.12740 & 4.29080 & 0.09880 & 0.96192 & 1.00000 & 16.00000 & 16.00000 & 0.12500 & 0.25372 & 81.92900 & 2.66660 & 99.00000 & 3.99850 & 77.910 \\
\hline 0.01863 & 0.54361 & 0.56224 & 0.03427 & 0.96687 & 11.00000 & 13.00000 & 3.00000 & 4.00000 & 0.01606 & 5549.90000 & 1.33330 & 4.00000 & 0.09636 & 81.733 \\
\hline
\end{tabular}

\begin{tabular}{|c|c|c|c|c|c|}
\hline 16 & 17 & 18 & 19 & 20 & 21 \\
\hline 1.000000 & $0.00 \mathrm{knOa}$ & G.onoun & noften & 0.093000 & oromon \\
\hline l.menoo & 0.00000 & 000000 & (5.000000 & a coevero & a.omow \\
\hline $1.0 \% 200$ & 0.00000 & $0.336 k 0)$ & 0.00000 & 0000000 & $00 t h 00$ \\
\hline I. 06000$)$ & ช.36600 & $0.000 \%$ & nonomo & a.0.00000 & 0.001400 \\
\hline $0.0100 \%)$ & 600000 & 0.00000 & $1.000 \mathrm{kHO}$ & 1).06000 & to $00 \mathrm{cos})$ \\
\hline OCONOW & 0.000000 & $0.00 \times 0$ & 1.000000 & 0.064000 & $(0.0 \mathrm{CH})(\mathrm{NO})$ \\
\hline 1.00000 & 0.0\%OWO & $0.000 x+4$ & $0.06 \% 00$ & o.jotuma & 0.6) \\
\hline 1.600000 & 0.00000 & (6.00000) & 0.000000 & 0,00090 & 0.00000 \\
\hline 160000 & 0.00000 & boun:s0 & 0.00000 & $0(30000$ & 0.000006 \\
\hline I. & $0.00 \mathrm{ck}$ ) & o.opkina & ondron & 0.000k & 0.000000 \\
\hline 1 .0000k & 0.00000 & b.schen & nockono & 0.100000 & $0.000 \mathrm{NO}$ \\
\hline 1,00000 & 0.00000 & $0.06 \mathrm{KH})$ & $0.000 \times 00$ & $0.00000)$ & 0.000000 \\
\hline $1006(h)$ & 0.00006 & o.ooteno & (nowho & $0.0(x)=0$ & 0.000010 \\
\hline 0.00000 & 0.00000 & 0.00000 & 1.8000000 & 0.06000 & 0.00000 \\
\hline $1.0 \times 000$ & 0.00000 & $0.00(\mathrm{~m})$ & $0.000 \times 30$ & $0.064,10$ & $0.60 \times(0)$ \\
\hline 1806000 & (1.06)(k3) & $0.0 \% \times O$ & (o.0\%om? & $00 \mathrm{FOCO}$ & 0.00000 \\
\hline 1.00000 & 0.60000 & 0.00000 & o. Orout & 0.00000 & oowno \\
\hline
\end{tabular}




\begin{tabular}{|c|c|c|c|c|c|}
\hline ] & 2 & 3 & 4 & 5 & 6 \\
\hline 0.19322 & 0.66292 & 0.81166 & 0.29147 & 0.81674 & 4,00000 \\
\hline 0.12833 & 0.30596 & 0.34620 & 0.41943 & 0.88377 & 7.00000 \\
\hline 0.23529 & 0.40886 & 0.61080 & 0.57548 & 0.66939 & 1.00000 \\
\hline 0.06450 & 0.19797 & 0.23980 & 0.32581 & 0.82557 & 0.00000 \\
\hline 0.00625 & 0.78755 & 0.79380 & 0.00794 & 0.99212 & 0.00000 \\
\hline 0.39088 & 1.44750 & 1.61900 & 0.27003 & 0.89408 & 1.00000 \\
\hline 0.17717 & 0.43967 & 0.54705 & 0.40296 & 0.80371 & 5.00000 \\
\hline 0.46979 & 2.34170 & 2.65740 & $0.2006 \mathrm{t}$ & 0.88121 & 0.00000 \\
\hline 0.47712 & 2.55580 & 2.70380 & 0.18667 & 0.94529 & 0.00000 \\
\hline 0.16083 & 1.25520 & 1.35130 & 0.12812 & 0.92887 & 3.00000 \\
\hline 0.29678 & 1.77160 & 1.85700 & 0.16751 & 0.95403 & 0.00000 \\
\hline 0.16612 & 2.49220 & 2.59420 & 0.06665 & 0.96069 & 4.00000 \\
\hline 0.65619 & 6.46290 & 6.57750 & 0.10153 & 0.98258 & 0.00000 \\
\hline 0.13887 & 1.00880 & 1. 10270 & 0.13765 & 0.91486 & 0.00000 \\
\hline 0.28524 & 3.40400 & 3.54150 & 0.08379 & 0.96117 & 1.00000 \\
\hline $0.44 ! 05$ & 4.09810 & 4. 16030 & 0.10762 & 0.98505 & 0.00000 \\
\hline 0.04156 & 0.47499 & 0.51530 & 0.08749 & 0.92178 & 15.00000 \\
\hline 0.03897 & 0.49133 & 0.53030 & 0.07932 & 0.92651 & 0.00000 \\
\hline 0.24647 & 1.99750 & 2.10970 & 0.12338 & 0.94684 & 2.00000 \\
\hline 0.31595 & 2.18740 & 2.37820 & 0.14444 & 0.91976 & 0.00000 \\
\hline \multicolumn{6}{|c|}{ Table 2. The validation data (actua! values). } \\
\hline 16 & 17 & 1.8 & 10) & 20 & 3 \\
\hline 1.00000 & $0.00 \%$ & 0,00000 & $000(900)$ & 0.00000 & $\operatorname{ann}(x)$ \\
\hline $100 \% 0$ & 0,00000 & 0.00000 & 0.00900 & 0.000000 & $0.0 \mathrm{mon}(\mathrm{t})$ \\
\hline 0.000013 & onomon & ootono & 1 nomot & nOMNOH & 0.00000 \\
\hline 0.00000 & ackoopo & 00100900 & 100000 & 00000010 & 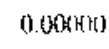 \\
\hline 1000000 & 0.0000 & 000000 & botouon & 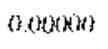 & 0.1360600 \\
\hline 1.00000 & $\theta \sin (a)$ & 0.00000 & 0.00000 & 0.00 (kin & 0.00040 \\
\hline $1000 \times 30$ & 0.00000 & o.00000] & $0.600 t) 0$ & 0.00000 & 000000 \\
\hline 100000 & 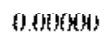 & 0.00000 & arowert & a (भค) & $0.0 \cos 10$ \\
\hline 1.000000 & n.thoroto & $0.000 \%$ & 0.60000 & $0.00 \times 120$ & 0.60000 \\
\hline $1.000 \times 0$ & 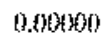 & 0.000000 & 0.000003 & ariforio & 0.000010 \\
\hline 100000 & O.,Whe & ockonos & $0.00 \times 10$ & овкин) & 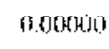 \\
\hline 1.00000 & 0.00000 & 0.000000 & a.conowo & 0.00 inn? & $0000 \times 10$ \\
\hline 1.00000 & 000000 & 0.00000 & a.oknth & о.он()ㅡㅇ & 0 oflotot \\
\hline 1.000000 & Denono & 0.000030 & 0.100000 & o.moto & 0.0000 \\
\hline 1.00000 & 000000 & onthoo & 0.00000 & 0.99000 & 0.00000 \\
\hline 1.000000 & $0.00000)$ & 0.00000 & 0.00000 & $0.00 \times(x)$ & 0.00000 \\
\hline a.oMono & 0.000013 & 0.00000 & f. 00000 & $0.0(x) m$ & $0.00 \times 10$ \\
\hline 1,00000 & Dopono & $0.00 \% 00$ & 0.00000 & 0.000060 & 0.00000 \\
\hline 1.00600 & 0.00000 & $0.000 \mathrm{BO}$ & 1).000100 & o.nogoo & $0.00 \times 30$ \\
\hline
\end{tabular}

$\begin{array}{rrrr}7 & 8 & 9 & 10 \\ 7.00000 & 4.00000 & 1.25000 & 0.04119 \\ 12.00000 & 6.00000 & 1.33330 & 0.07902 \\ 5.00000 & 5.00000 & 0.40000 & 0.06192 \\ 1.00000 & 2.00000 & 0.50000 & 0.01531 \\ 5.00000 & 6.00000 & 0.16667 & 0.01962 \\ 10.00000 & 10.00000 & 0.20000 & 0.10606 \\ 7.00000 & 3.00000 & 2.00000 & 0.03205 \\ 10.00000 & 11.00000 & 0.09091 & 0.26227 \\ 12.00000 & 13.00000 & 0.07692 & 0.12257 \\ 11.00000 & 9.00000 & 0.44444 & 0.05357 \\ 9.00000 & 10.00000 & 0.10000 & 0.20973 \\ 14.00000 & 11.00000 & 0.45455 & 0.06228 \\ 26.00000 & 27.00000 & 0.03704 & 0.57548 \\ 5.00000 & 6.00000 & 0.16667 & 0.03399 \\ 18.00000 & 18.00000 & 0.11111 & 0.12983 \\ 18.00000 & 19.00000 & 0.05263 & 0.20093 \\ 16.00000 & 2.00000 & 8.00000 & 0.03013 \\ 4.00000 & 5.00000 & 0.20000 & 0.04180 \\ 10.00000 & 9.00000 & 0.33333 & 0.06891 \\ 8.00000 & 9.00000 & 0.11111 & 0.07196\end{array}$

11
7764.20000
1437.10000
5100.60000
4535.60000
93.63900
1254.50000
11800.00000
1165.10000
408.97000
991.11000
1289.50009
1128.10000
307.51000
2197.10000
320.50000
953.26000
10927.00000
1669.60000
1761.20000
1476.10000

12
1.66660
3.33330
1.66660
1.33330
1.33330
2.33330
3.00000
2.00000
2.33330
1.00000
3.66660
1.00000
2.33330
2.33330
1.33330
2.33330
2.00600
1.33330
3.33330
1.66660

9.00000
16.00000
12.00000
4.00000
7.00000
33.00000
9.00000
46.00000
64.00000
17.00000
46.00000
21.00000
513.00000
11.00000
97.00000
114.00000
6.00000
7.00000
28.00000
21.00000




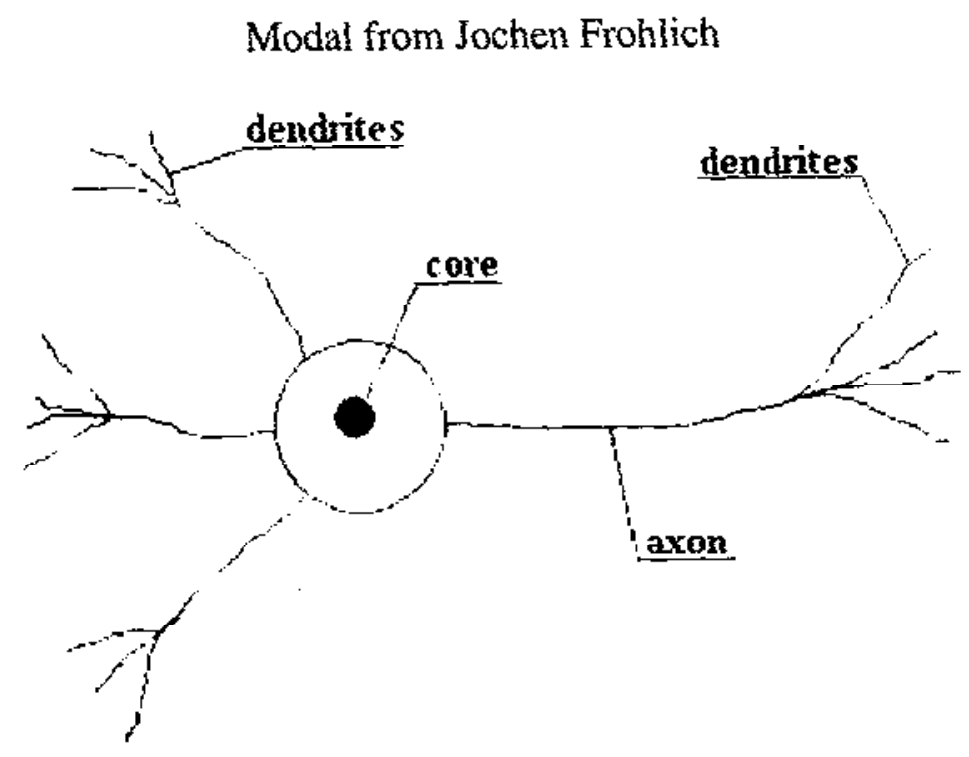

Figure 1. Biological Network

Dendrites- accept the inputs.

Core- processes the input.

Axon-turns the into outputs 
Figure 2. The JOONE editor.

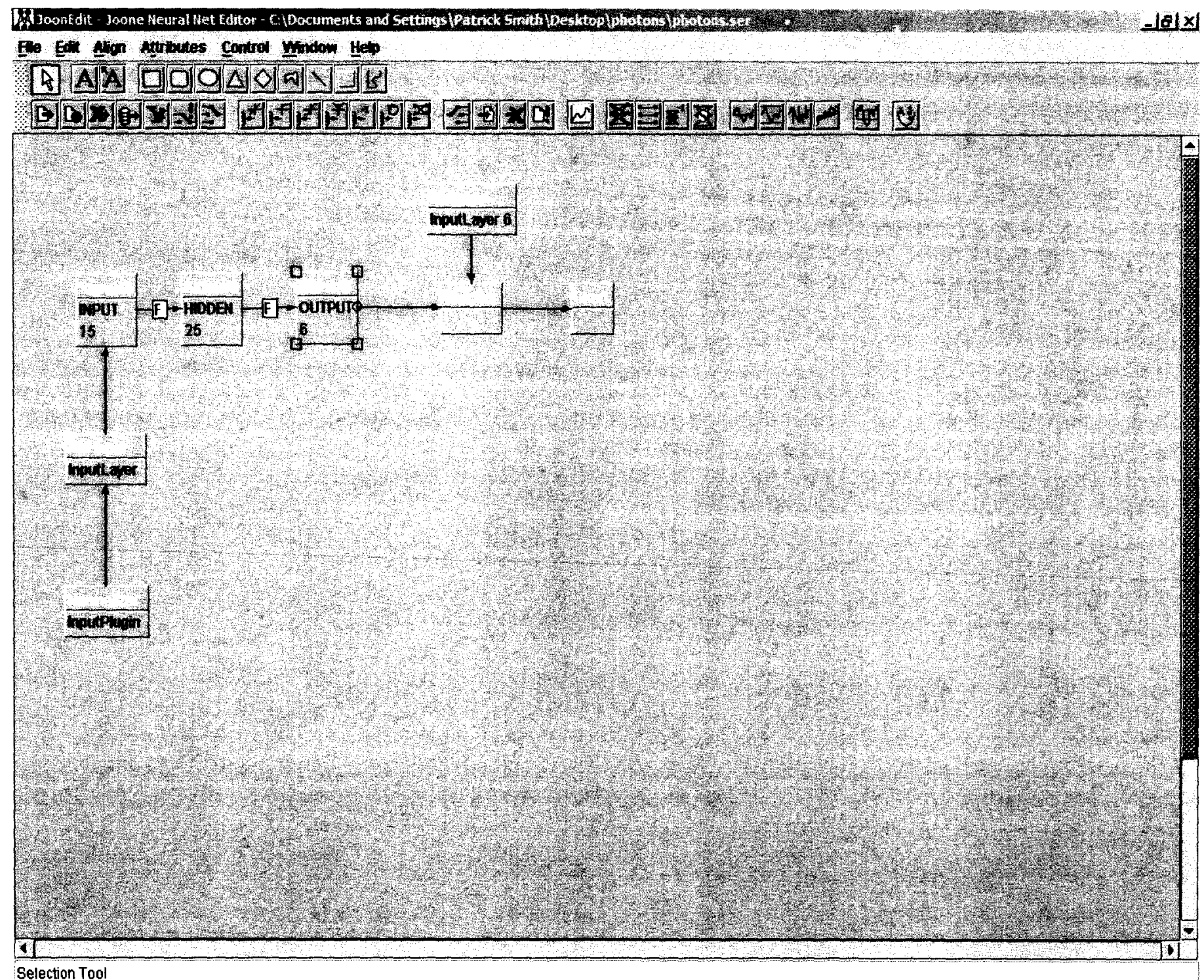


Figure 3. The JOONE plot of an unsuccessful 15-25-6 multi-payer perceptron train net.

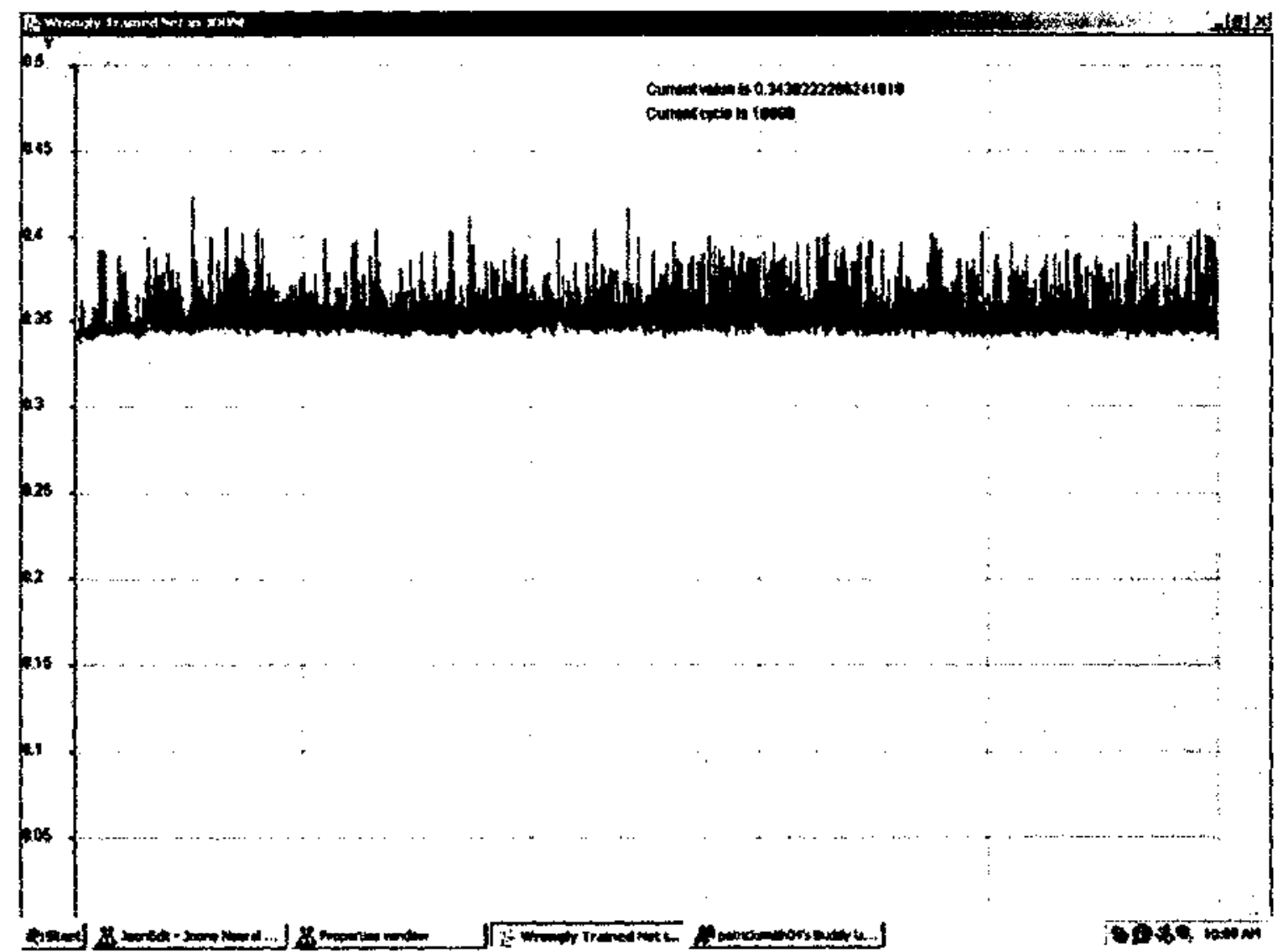


Figure 4. The CJNN output plot.

\section{Error}

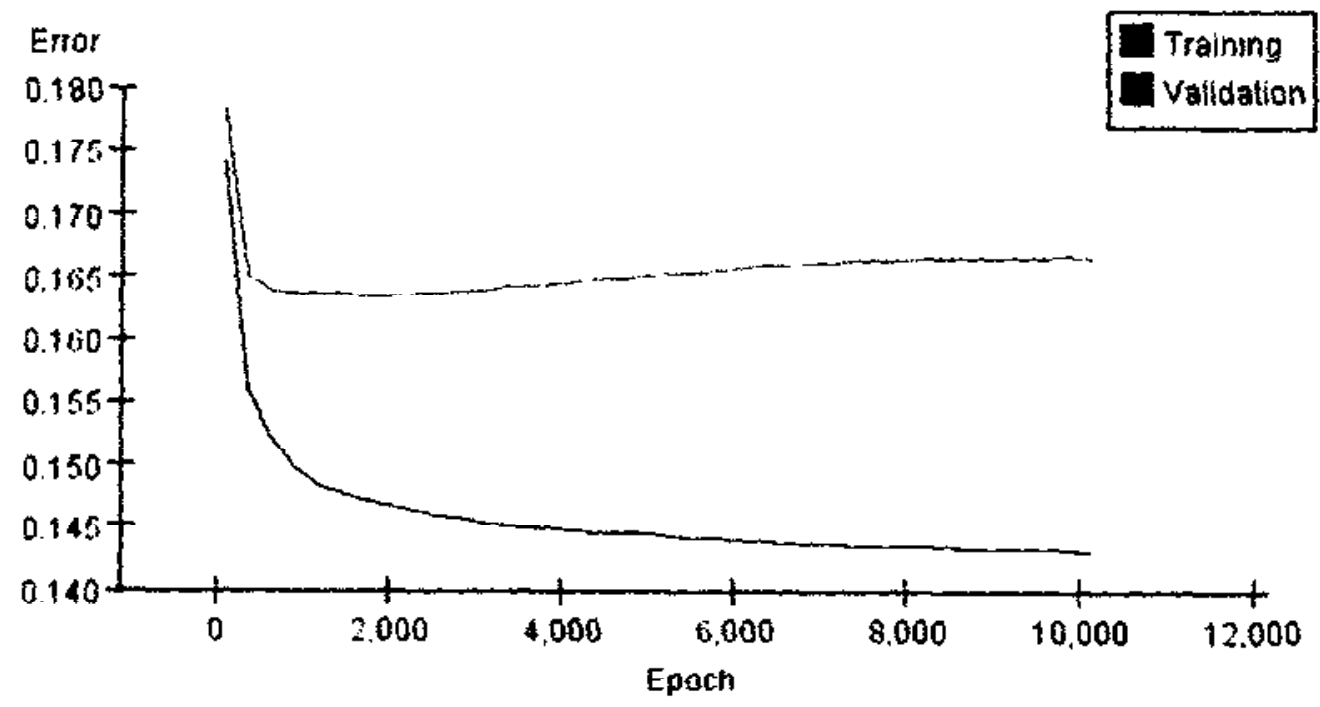


FIGURE 5. plots comparing the desired output with the resulting output

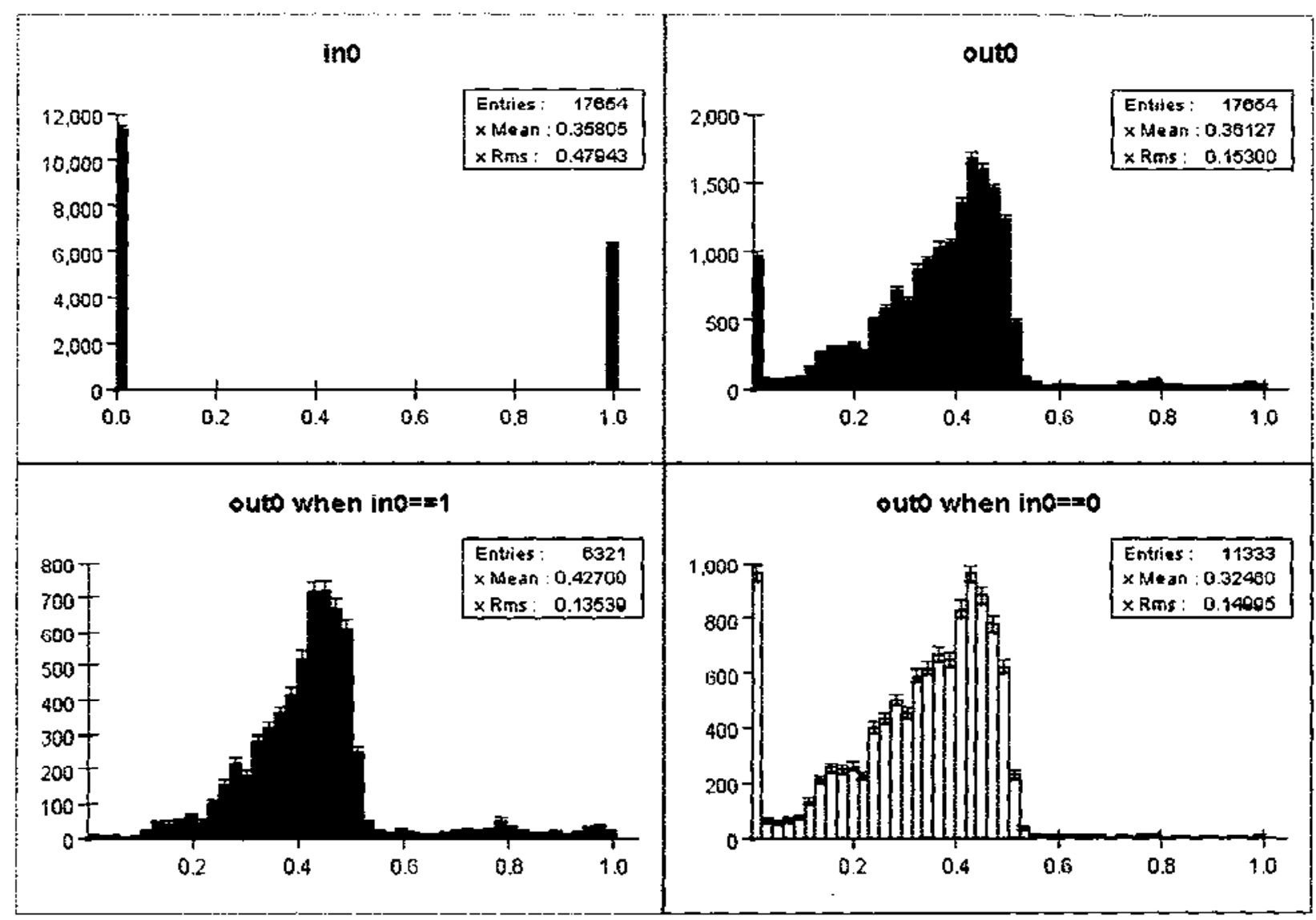

